\title{
AN ERGODIC SUPER-PROPERTY OF BANACH SPACES DEFINED BY A CLASS OF MATRICES
}

\section{A. BRUNEL, H. FONG ${ }^{1}$ AND L. SUCHESTON ${ }^{1}$}

ABSTRACT. A matrix $\left(a_{n i}\right)$ is called an $R$-matrix if (A) $\Sigma_{i} a_{n i} \nrightarrow 0$, and (B) $\lim _{n} a_{n i}=0$ for each $i$. A Banach space $X$ is called $R$-ergodic if for each isometry $T$ and each $x \in X$, there is an $R$-matrix $\left(a_{n i}\right)$ such that $\Sigma_{i} a_{n i} T^{i} x \stackrel{\mathrm{w}}{\rightarrow}$ (converges weakly). Given two Banach spaces $F$ and $X$, write $F$ fr $X$ if for each finite-dimensional subspace $F^{\prime}$ of $F$ and $\epsilon>0$, there is an isomorphism $V$ from $F^{\prime}$ onto a subspace of $X$ such that $|\|x\|-\|V x\||<\epsilon$ for each $x \in F^{\prime}$ with $\|x\| \leq 1$. $X$ is called super-R-ergodic if $F$ is $R$-ergodic for each $F$ fr $X$.

Theorem. $X$ is super-R-ergodic if and only if $X$ is super-reflexive.

The proof is based on the following:

Theorem. Let $T$ be a linear operator on $X,\left(a_{n i}\right)$ a matrix satisfying (A), $x \in X$ such that $\Sigma_{i} a_{n i} T^{i} x \underline{w} \bar{x}$. Then there is a constant a such that $(x-a \bar{x}) \in \overline{(I-T) X}$.

A matrix $\left(a_{n i}\right)$ with real terms is called an $R$-matrix iff it satisfies the following conditions:

$$
\begin{gathered}
\sum_{i} a_{n i} \nrightarrow 0 \text { as } n \rightarrow \infty \\
\lim _{n} a_{n i}=0 \text { for each } i .
\end{gathered}
$$

Condition (A) means that $\Sigma_{i} a_{n i}$ exists for each $n$ and the sequence $\left(\Sigma_{i} a_{n i}\right)$ either diverges or converges to a limit different from zero.

A Banach space $X$ is called $R$-ergodic iff for each isometry $T$ and each $x \in X$ there exists an $R$-matrix $\left(a_{n i}\right)$ such that $\Sigma_{i} a_{n i} T^{i} x$ converges weakly. It is shown that $X$ is super-R-ergodic if and only if it is super-stable (equivalently, super-reflexive). Since $R$-ergodicity is clearly implied by

Presented to the Society, February 28, 1974; received by the editors June 11, 1973 and, in revised form, March 1, 1974.

AMS (MOS) subject classifications (1970). Primary 47A35; Secondary 46B10.

Key words and phrases. $R$-matrices, super-properties, $R$-ergodic, reflexive, stable.

1 This work is supported by the National Science Foundation, grant GP 34118. 
ergodicity, this is an improvement over the results of [1] and [2], to which the present theorem is reduced by an ergodic argument. It is further observed that a Banach space $X$ is reflexive if and only if for each bounded sequence $\left(x_{i}\right)$ in $X$ there is an $R$-matrix $\left(a_{n i}\right)$ such that $\Sigma_{i} a_{n i} x_{i} \stackrel{w}{\rightarrow}(\stackrel{w}{\rightarrow}$ means: converges weakly).

1. A real Banach space $X$ is given, with elements $x, y, \cdots$. Sequences of real numbers are denoted by $a=\left(a_{i}\right), b=\left(b_{i}\right)$, etc. $S$ is the set of all sequences $\left(a_{i}\right)$ such that $a_{i} \neq 0$ for only finitely many indices $i$. Whenever we write $\Sigma a_{i} x_{i}$, we tacitly assume that the summation makes sense in $X$. Given an operator $T$, we write $A_{n}(T)$ or $A_{n}$ for the operator $(1 / n)\left(T^{0}+T^{1}+\cdots+T^{n-1}\right)$. The following theorem relates the local behavior of $\Sigma_{i} a_{n i} T^{i}$ to the local behavior of $A_{n}(T)$. An A-matrix is one satisfying the condition (A).

Theorem 1. Let $T$ be a linear operator in $X,\left(a_{n i}\right)$ an A-matrix, and $x$ in $X$ such that $\Sigma_{i} a_{n i} T^{i} x \stackrel{\mathrm{w}}{\rightarrow} \bar{x}$. Then there exists a constant a such that $(x-\alpha \bar{x})$ belongs to the closure of $(I-T) X$. a may be chosen equal to 1 if $\lim _{n} \Sigma_{i} a_{n i}=1 . A_{n}(x-\alpha \bar{x}) \rightarrow 0$ if

$$
\sup _{n}\left\|A_{n}(T)\right\|<\infty \text { and } T^{n} / n \rightarrow 0 \text { strongly. }
$$

Proof. We first prove the theorem under the additional assumption that $\Sigma_{i} a_{n i}=1$ and $\left(a_{n i}\right)_{i} \in S$ for each $n$. Let a map $\phi: S \rightarrow X$ be defined by $\phi(a)=\Sigma a_{i} T^{i} x$. If $\Sigma_{i=0}^{n-1} b_{i}=0$, then

$$
\begin{aligned}
\sum_{i=0}^{n-1} b_{i} T^{i}= & b_{0}(I-T)+\left(b_{0}+b_{1}\right)\left(T-T^{2}\right)+\left(b_{0}+b_{1}+b_{2}\right)\left(T^{2}-T^{3}\right) \\
& +\cdots+\left(b_{0}+b_{1}+\cdots+b_{n-1}\right)\left(T^{n-1}-T^{n}\right) \\
= & P(T)(I-T),
\end{aligned}
$$

where $P(T)$ is a polynomial in $T$. Therefore for each $b \in S$,

$$
\phi(b) \in(I-T) \phi(S)
$$

if $\Sigma b_{i}=0$. This remark is applied to the sequence $\left(b_{i}\right)$ defined by $b_{0}=$ $a_{n 0}-1, b_{i}=a_{n i}$ for $i>0, n$ fixed. It follows that for each $n$ there exists a $y_{n} \in \phi(S)$ such that $\Sigma_{i} a_{n i} T^{i} x-x=y_{n}-T y_{n}$. Therefore $\bar{x}-x$ belongs to the weak closure of $(I-T) \phi(S)$, identical (Hahn-Banach) with the strong closure $\left(\overline{I-T) \phi(S)}\right.$. Assume $(1) ; A_{n}(x-\bar{x}) \rightarrow 0$ follows, by approximation, 
from convergence to zero of expressions of the form $T^{n} y / n, y \in X$.

Now consider the general case. Let $\mathbf{K}$ be the set of all strictly increasing sequences of nonnegative integers. We may assume that there is a positive number $\beta$ such that $\Sigma_{i} a_{n i}>\beta$ for each $n$. (If necessary, replace $\left(a_{n i}\right)$ by $\left(a_{k_{n}, i}\right),\left(k_{n}\right) \in \mathbf{K}$; if necessary, change signs.) Let $x_{i}=T^{i} x,\left(k_{n}\right) \in \mathbf{K}$ be such that $\left|\Sigma_{i>k_{n}} a_{n i}\right|<1 / n$ and $\left\|\Sigma_{i>k_{n}} a_{n i}{ }_{i}\right\|<1 / n$. Let $d_{n i}=a_{n i}$ if $i \leq k_{n}$; $d_{n i}=0$ for $i>k_{n}$. Then $\left(d_{n i}\right)$ is an $A$-matrix, $\left(d_{n i}\right)_{i} \in S$ for each $n$ and $\beta \leq$ $\lim \inf _{n} \Sigma_{i} d_{n i}=d \leq \infty$. The last lim inf may be assumed to be limit, because $\left(d_{n i}\right)$ may again be replaced by a submatrix. Set $b_{n i}=d_{n i} / \Sigma_{j} d_{n j}$ for all $n$ and $i$. Then $\left(b_{n i}\right)$ is an $A$-matrix, and for each $n,\left(b_{n i}\right)_{i} \in S$ and $\Sigma_{i} b_{n i}=1$. Furthermore, $\Sigma_{i} d_{n i} x_{i} \stackrel{w}{\rightarrow} \bar{x}$ implies that $\Sigma_{i} b_{n i} x_{i} \stackrel{w}{\rightarrow} \alpha \bar{x}$, with $a=1 / d$. The first part of the proof now applies to the matrix $\left(b_{n i}\right)$, showing that $(x-a \bar{x})$ $\in \overline{(I-T) X} \cdot \vdash$

Corollary 1 (Ergodic theorem of Yosida-Kakutani; cf. [4, p. 661]). Assume (1) and let $x \in X$ be such that $A_{k_{n}} \underset{\text { w }}{\rightarrow} \bar{x}$ for some $\left(k_{n}\right) \in \mathbf{K}$. Then $A_{n} x \rightarrow \bar{x}$.

Proof. (I $-T) A_{k_{n}} x$ converges to zero (cancellation properties of Cesàro averages) and also converges weakly to $(I-T) \bar{x}$. Therefore $T \bar{x}=\bar{x}$. Write $A_{k_{n}} x=\Sigma_{i} a_{n i} T^{i} x$; then $\left(a_{n i}\right)$ is an $A$-matrix with $\Sigma_{i} a_{n i}=1$; thus $A_{n}(x-\bar{x})=$ $A_{n} x-\bar{x} \rightarrow 0 . \vdash$

Given two Banach spaces $X,\|\|$ and $F, \|, F$ is said to be finitely representable in $X$, in symbols $F \mathrm{fr} X$, iff for each finite-dimensional subspace $F^{\prime}$ of $F$ and each number $\epsilon>0$, there is an isomorphism $V$ of $F^{\prime}$ into $X$ such that ||$x|-\|V x\|| \leq \epsilon$ for each $x$ in the unit ball $U_{F^{\prime}}$ of $F^{\prime}$. If $P$ is a property of Banach spaces, say that $X$ is super- $P$ iff $F \operatorname{fr} X$ implies that $F$ is $P . X$ is called $R$-ergodic iff for each isometry $T$ on $X$ and each $x \in X$ there exists an $R$-matrix $\left(a_{n i}\right)$ such that $\Sigma_{i} a_{n i} T^{i} x$ converges weakly. A bounded sequence $\left(x_{n}\right)$ in $X$ is called stable iff there is an element $\bar{x}$ such that

$$
\left\|(1 / n)\left(x_{k_{1}}+\cdots+x_{k_{n}}\right)-\bar{x}\right\| \rightarrow 0
$$

uniformly in the set K. $X$ is called stable iff every bounded sequence contains a stable subsequence. 
Theorem 2. A Banach space is super-stable if (and only if) it is superR-ergodic.

Proof. In [1] and [2] the same result is proved with ergodicity (i.e., strong convergence of $A_{n}(T)$ for each isometry $T$ ) instead of $R$-ergodicity. Assume that $Y$ is super- $R$-ergodic and let $X$ fr $Y$. We follow the notation and argument in [1]. From a sequence $\left(x_{n}\right)$ in $U_{X}$ we wish to extract a stable subsequence. First obtain a subsequence $\left(e_{n}\right)$ of $\left(x_{n}\right)$ such that the space $F$ generated by $\left(e_{n}\right)$ and a new norm || satisfies $F$ fr $X$, and also $(1 / n)\left(e_{0}+\cdots+e_{n-1}\right) \rightarrow$ in $F$ implies that $\left(e_{n}\right)$ contains a subsequence stable in $X$ [1, Proposition 3]. The shift $T$ on $\left(e_{n}\right)$ is an isometry, and the norm || is of type (IS), or "invariant under spreading" of the $e_{n}$ 's [1, Lemma 1]. The (IS) property implies that, in ergodic terminology, the "tail" space is equal to the "invariant" space (strictly speaking, the space of the invariant elements). We mean by this that $x \in \bigcap_{k} T^{k} F$ if and only if $T x=x[1$, Lemma 4]. Now $F$ fr $X$ fr $Y$ implies $F$ fr $Y$; hence $F$ is $R$-ergodic: There exists an $R$-matrix $\left(a_{n i}\right)$ and an element $\bar{e}$ such that $x_{n}=\Sigma_{i} a_{n i} e_{i} \stackrel{\mathrm{w}}{\rightarrow} \bar{e}$ in $F$. The property (B) of the matrix implies that for each $k, \bar{e}$ belongs to the weak closure of $T^{k} F$, hence $\bar{e} \in T^{k} F$. Indeed, if $y_{n}=\Sigma_{i \geq k} a_{n i} e_{i}$, then $y_{n} \in T^{k} F$ for each $n$ and $\left|x_{n}-y_{n}\right| \rightarrow 0$, implying $y_{n} \stackrel{\mathrm{w}}{\rightarrow} \bar{e}$. Therefore $T \bar{e}=\bar{e}$. From Theorem 1 we obtain that $A_{n} e_{0} \rightarrow \bar{e}$ in $F$. Therefore $\left(e_{n}\right)$ has a subsequence stable in $X$. Since $\left(x_{n}\right)$ is arbitrary, $X$ is stable; since $X$ is an arbitrary space satisfying $X$ fr $Y, Y$ is super-stable. -

$R$-matrices appear in the following characterization of reflexivity.

Theorem 3. A Banach space $X$ is reflexive if and only if for each sequence $\left(x_{n}\right)$ in $U_{X}$ there is an R-matrix $\left(a_{n i}\right)$ such that $\Sigma_{i} a_{n i} x_{i}$ converges weakly.

Proof. The only if part follows at once from the known characterization of reflexivity in terms of weak sequential compactness of $U_{X}$. The if part is a simple consequence of a deep theorem of PeXczyński [6]; we learned about this theorem from Professor W. Johnson. Assume that $X$ is not reflexive. By [6] there exists a bounded basic sequence $\left(x_{i}\right)$ and a bounded linear functional $f$ such that $\lim \sup _{i} f\left(x_{i}\right)>0$. Passing to a subsequence if necessary, we may assume that there is an $\alpha>0$ with $f\left(x_{i}\right) \geq \alpha$ for each $i$. Set $y_{i}=x_{i} / f\left(x_{i}\right)$; then $\left(y_{i}\right)$ is a bounded basic sequence and $f\left(y_{i}\right)=1$ for each $i$. Assume that there is an $R$-matrix $\left(a_{n i}\right)$ such that $\Sigma_{i} a_{n i} y_{i} \stackrel{\mathrm{w}}{\rightarrow} \bar{y}$; let $\bar{y}=$ $\Sigma_{i} a_{i} y_{i}$. The continuity of the coefficient functionals and condition (B) 
imply that $a_{i}=\lim _{n} a_{n i}=0$ for each $i$. Thus $\bar{y}=0$. Now

$$
0=f(\bar{y})=\lim _{n} f\left(\sum_{i} a_{n i} y_{i}\right)=\lim _{n} \sum_{i} a_{n i} f\left(y_{i}\right)=\lim _{n} \sum_{i} a_{n i},
$$

which contradicts condition (A). $\vdash$

Remarks。(1) $A$-matrices and $R$-matrices have the following properties: Given a bounded sequence $\left(x_{i}\right)$, if there is an $A$-matrix (resp., $\left.R-\right)\left(a_{n i}\right)$ such that $\Sigma_{i} a_{n i} x_{i} \stackrel{\mathrm{w}}{\rightarrow}$, then there is another $A$-matrix (resp., $\left.R_{-}\right)\left(b_{n i}\right)$ such that $\Sigma_{i} b_{n i} x_{i} \rightarrow$ strongly. This easily follows from Mazur's theorem [4, p. 422, Corollary 14].

(2) Condition (A) (and trivially, condition (B)) cannot be dispensed with in Theorem 3: The space $c_{0}$ is alternate signs Banach-Saks: i.e., each bounded sequence contains a subsequence $\left(y_{i}\right)$ with

$$
(1 / n)\left(y_{1}-y_{2}+\cdots+(-1)^{n-1} y_{n}\right) \rightarrow 0
$$

[3, Proposition 3.1], but $c_{0}$ is not reflexive. However, $(A)$ and (B) may be replaced by the following slightly weaker set of conditions $\left(R^{\prime}\right)$ :

(C) $\lim _{n} a_{n i}=a_{i}$ exists for each $i$ and $\Sigma_{i} a_{i}$ converges, and

(D) $\Sigma_{i} a_{n i} \nrightarrow \Sigma_{i} a_{i}$, where (D) means that $\Sigma_{i} a_{n i}$ exists for each $n$ and the sequence $\left(\Sigma_{i} a_{n i}\right)$ either diverges or converges to a limit different from $\Sigma_{i} a_{i}$

Theorem 3 together with the proof given above remain valid if the condition $(R)$ is replaced by the condition $\left(R^{\prime}\right)=(C)+(D)$. Thus amended, Theorem 3 includes the theorems of Nishiura and Waterman [5] and Waterman [8].

(3) A variant of the proof of Theorem 2 may be based on Remark (1) and Theorem 3, as follows: From a sequence $\left(x_{i}\right)$ in $U_{X}$ extract a subsequence $\left(e_{i}\right)$ and form the space $F$ as in Theorem 2; by $R$-ergodicity and Remark (1), an $R$-matrix $\left(a_{n i}\right)$ exists such that $\Sigma_{i} a_{n i} e_{i} \rightarrow$ in $F$. Using an argument similar to that of $[1$, Proposition 3$]$, one obtains a subsequence $\left(y_{i}\right)$ of $\left(e_{i}\right)$ and an $R$-matrix $\left(b_{n i}\right)$ for which $\Sigma_{i} b_{n i} y_{i} \rightarrow$ in $X$. By Theorem $3, X$ is reflexive; since $X$ fr $Y$ was arbitrary, $Y$ is super-reflexive.

\section{REFERENCES}

1. A. Brunel and L. Sucheston, On B-convex Banach spaces, Math. Systems Theory 7 (1973/74), 294-299.

2. - Sur quelques conditions équivalentes à la super-réflexivité dans les espaces de Banach, C. R. Acad. Sci. Paris Sér A-B 275 (1972), A993-A994. MR 47 \#7389. 
3. A. Brunel and L. Sucheston, On J-convexity and some ergodic super-properties of Banach spaces, Trans. Amer. Math. Soc. (to appear).

4. N. Dunford and J. T. Schwartz, Linear operators. I: General theory, Pure and Appl. Math., vol. 7, Interscience, New York, 1958. MR 22 \#3302.

5. T. Nishiura and D. Waterman, Reflexivity and summability, Studia Math. 23 (1963), 53-57. MR 27 \#5107.

6. A. Pelczyński, A note on the paper of I. Singer "Basic sequences and reflexivity of Banach spaces," Studia Math. 21 (1961/62), 371-374. MR 26 \#4156. 7. I. Singer, Bases in Banach spaces. I, Die Grundlehren der math. Wissenschaften, Band 154, Springer-Verlag, Berlin and New York, 1970.

8. D. Waterman, Reflexivity and summability. II, Studia Math. 32 (1969), 6163. MR $39 \# 7399$.

DEPARTMENT OF MATHEMATICS, UNIVERSITY OF PARIS VI, 9 QUAI ST. BERNARD, 75005, PARIS, FRANCE (Current address of A. Brunel)

DEPARTMENT OF MATHEMATICS, OHIO STATE UNIVERSITY, COLUMBUS, OHIO 43210 (Current address of L. Sucheston)

Current address (H. Fong): Department of Mathematics, Bowling Green State University, Bowling Green, Ohio 43403 